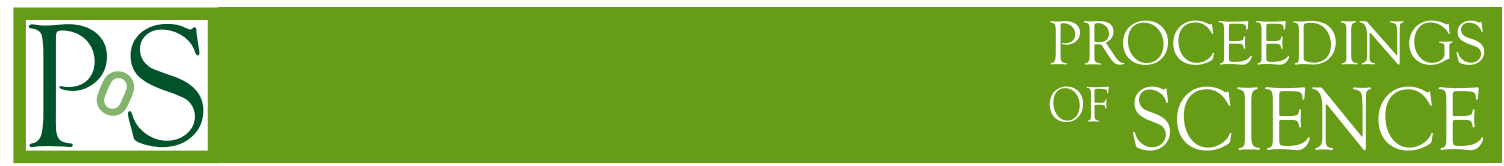

\title{
Searches for displaced and delayed signatures at the LHC
}

\author{
Rachel Rosten ${ }^{a, *}$ on behalf of the ATLAS and CMS Collaborations \\ ${ }^{a}$ Ohio State University, \\ 191 West Woodruff Ave, Columbus, OH 43210, USA \\ E-mail: rachel.rosten@cern.ch
}

A wide variety of physics models can give rise to particles with long lifetimes. While the ATLAS and CMS experiments at the LHC were designed primarily to identify promptly decaying particles, both collaborations host multiple analyses targeting the decay of hypothetical particles with macroscopic decay lengths. This proceeding considers two such analyses. The first, an ATLAS analysis, identifies pairs of displaced leptons which do not share a vertex. The second, a CMS analysis, targets clusters in the muon system endcap with high hit multiplicity. The strategy and results of each analysis are reported.

The Ninth Annual Conference on Large Hadron Collider Physics - LHCP2021

7-12 June 2021

Online

*Speaker 


\section{Introduction}

The ATLAS [1] and CMS [2] experiments at the LHC were designed with promptly decaying particles in mind. The resulting Standard Model (SM) leptons or hadronic showers will have their origin near the proton-proton interaction point. However, a variety of potential extensions to the SM give rise to particles capable of traveling some macroscopic distance within the detector before decaying to detectable SM daughters. This proceeding focuses on two searches for such long-lived particles (LLPs).

\section{Displaced Leptons in ATLAS}

Consider the decay of a slepton under the gauge-mediated supersymmetry breaking SUSY model. Here, the gravitino is nearly massless and is stable. A slepton decaying to its SM partner and a gravitino will produce the signature in ATLAS of an isolated lepton track missing inner hits in the Inner Detector (ID) [3]. Depending on the specific model, the reconstructed events may also have no significant missing transverse energy. Previous searches in ATLAS and CMS have focused on lepton tracks from displaced vertices [4] or on disappearing tracks resulting from the decay where the charged daughter has too little momentum for the track to be reconstructed $[5,6]$. Indeed, the best limits previously set on the chosen model hail from OPAL [7].

Reconstruction of the displaced leptons in this analysis relies on the large-radius tracking algorithm [8]. This algorithm, contrary to the standard one, allows for the reconstruction of tracks with large impact parameters $\left(d_{0}\right)$ and missing inner detector hits. Fig. 1 illustrates the sensitivity gain when comparing this algorithm, referred to in the figure as "extended tracking", and lepton reconstruction relying on standard tracking. The additional benefit from loosening impact parameter and hit requirements on the lepton identification algorithms themselves is also illustrated.

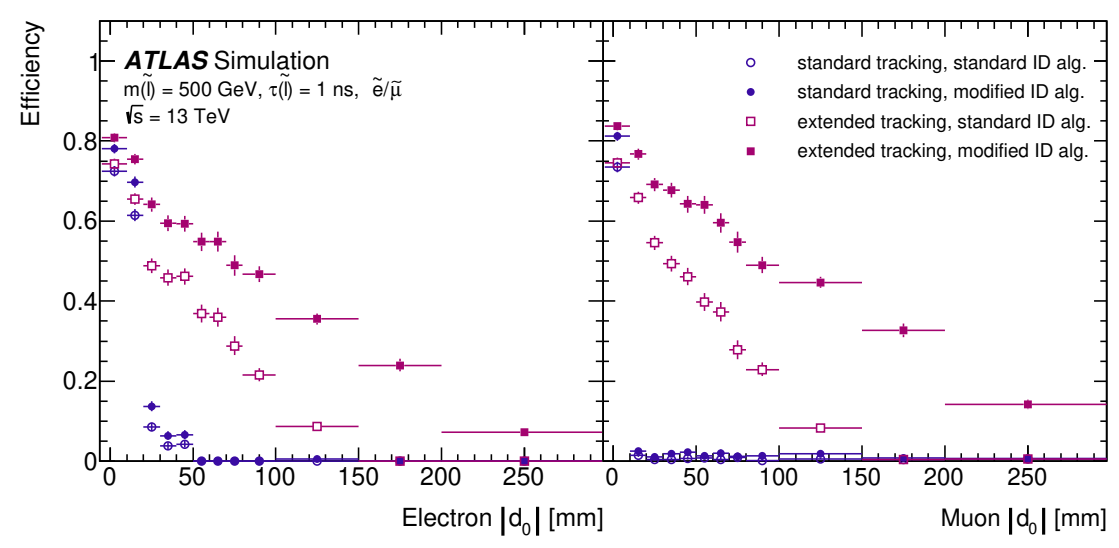

Figure 1: Electron (left) and muon (right) reconstruction efficiencies for standard and modified tracking and identification criteria [3].

The analysis strategy begins with selecting events with ATLAS triggers targeting muons reconstructed without relying on the ID as well as photon triggers, the latter being useful for selecting electrons. A requirement for two displaced lepton tracks with an opening angle $\Delta R>0.2$ 
is made, with the opening angle required to eliminate events resulting from interaction in the material of the ID. After application of a veto on cosmic ray muon events, the background estimate for all final state combinations of leptons is less than a single event.

No events were observed, consistent with the background prediction of less than one event. The analysis set limits on the lifetime and mass of the slepton, shown on the left-hand side of Fig. 2. The weaker limits for the stau are a result of the branching ratio of the tau to leptons. To illustrate the improvement over the previous best limits, set by OPAL, Fig. 2 includes on the right the OPAL and ATLAS limits for the smuon decay.
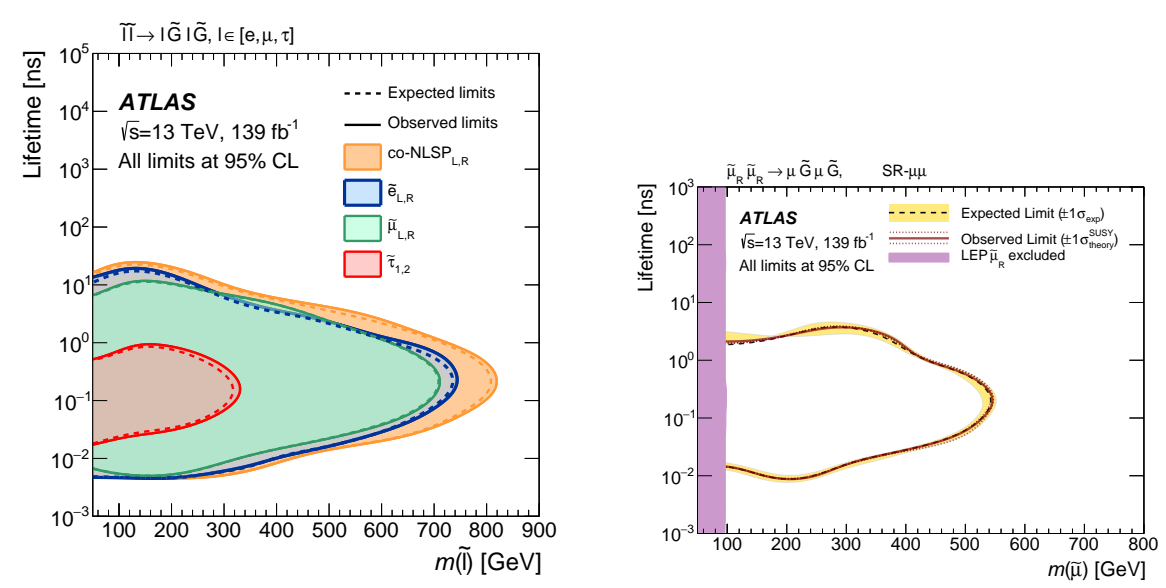

Figure 2: Limits for sleptons (left) and a comparison with the previous most stringent limit for smuons (right) [3].

\section{Long-lived Particles in the CMS Endcap Muon System}

Consider a simplified model where the SM Higgs boson decays to a pair of long-lived, neutral scalars, $S$, which in turn decay to pairs of SM particles. If this decay happens within the CMS Muon System (MS) EndCap (EC), the alternating layers of sensitive muon chambers and steel will act more as a sampling calorimeter than a tracking system. The decay of the $S$ in this region will not result in a pair of nicely reconstructed jets, for example, but instead in a cascade of hadrons and leptons which will leave many hits in the MS EC. Identifying isolated clusters of hits in this region is then a novel way of searching for LLP decays [9].

The analysis strategy begins by selecting events with a CMS missing energy trigger. This effectively requires that the Higgs boson recoils off of an initial state radiation jet. Offline, the trigger requirements translate to a $200 \mathrm{GeV}$ missing energy requirement and a requirement of at least one jet with $p_{\mathrm{T}}>50 \mathrm{GeV}$. The innermost $\phi$ rings of each station have the least amount of shielding and are prone to having more hits from SM activity, so clusters with any hits in these stations are rejected. The analysis vetoes on hard leptons to suppress backgrounds from $W$ and top production and suppresses the muon bremsstrahlung background, which tends to be forward, with a requirement that clusters satisfy $|\eta|>2.4$. A pre-existing clustering algorithm [10] is used to form clusters, while a custom algorithm to identify signal clusters provides a suppression factor of 3 for the remaining background. 
The most important variables for identifying good clusters are the number of hits in the cluster, $N_{\text {hits }}$, and the angle in $\phi$ between the cluster center and the missing transverse momentum, $\Delta \phi\left(\vec{p}_{\mathrm{T}}^{\text {miss }}\right.$, cluster $)$, both shown in Fig. 3. The reason for the latter cut is that the remaining background after all other cuts is primarily activity from vertices other than the primary vertex, activity that is independent of the missing transverse momentum.
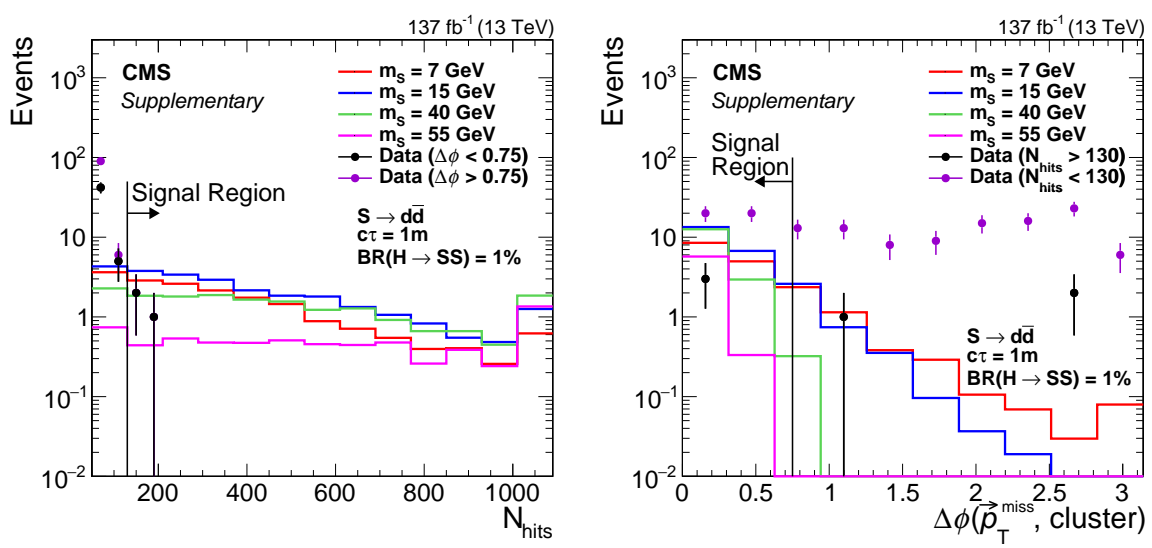

Figure 3: The most powerful cuts for identifying signal clusters are shown: the number of hits (left) and the angle between the cluster and missing transverse momentum (right) [9].

The final background prediction for this search is $2 \pm 1$ events, while three events were observed. Limits were set on the lifetime of the scalar, as seen in Fig. 4. The limits are the best yet of any analysis for the lightest scalars and competitive with ATLAS at other masses [11].
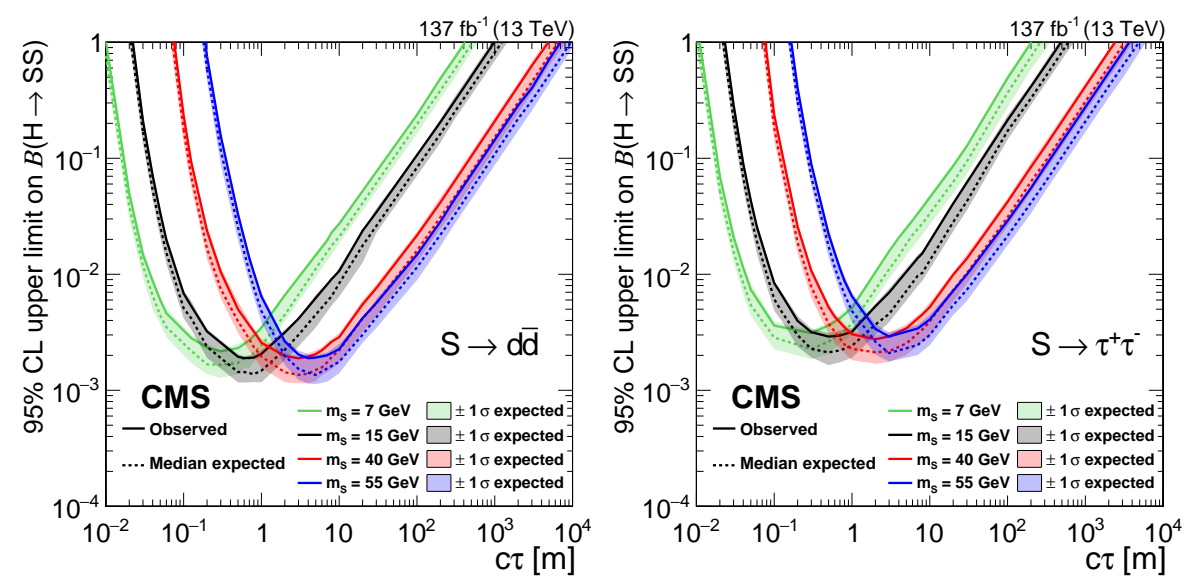

Figure 4: The limits set for a long-lived scalar decaying to down quarks (left) and tau leptons (right) [9].

\section{Summary}

While many searches for long-lived particles at both ATLAS and CMS experiments at the LHC focus on vertex reconstruction, both collaborations include LLP searches with alternative final states. This proceeding summarizes two recent results, one from each collaboration, looking for unique final states. While neither analysis found evidence of new physics, both set impressive limits and laid the foundation for the search to continue into Run 3. 


\section{References}

[1] ATLAS Collaboration, The ATLAS Experiment at the CERN Large Hadron Collider, JINST 3 (2008) S08003.

[2] CMS Collaboration, The CMS experiment at the CERN LHC, JINST 3 (2008) S08004.

[3] ATLAS Collaboration, Search for displaced leptons in $\sqrt{s}=13 \mathrm{TeV} p \mathrm{p}$ collisions with the ATLAS detector, Phys. Rev. Lett. 127 (2021) 051802 [hep-ex/2011.07812].

[4] ATLAS Collaboration, Search for displaced vertices of oppositely charged leptons from decays of long-lived particles in pp collisions at $\sqrt{s}=13 \mathrm{TeV}$ with the ATLAS detector, Phys. Lett. B 801 (2020) 135114 [hep-ex/1907. 10037].

[5] ATLAS Collaboration, Search for long-lived charginos based on a disappearing-track signature using $136 \mathrm{fb}^{-1}$ of p collisions at $\sqrt{\mathrm{s}}=13 \mathrm{TeV}$ with the ATLAS detector, ATLAS-CONF2021-015.

[6] CMS Collaboration, Search for disappearing tracks in proton-proton collisions at $\sqrt{s}=13$ TeV, Phys. Lett. B 806 (2020) 135502 [hep-ex/2004 . 05153].

[7] OPAL Collaboration, Searches for Gauge-Mediated Supersymmetry Breaking topologies in $e^{+} e^{-}$collisions at centre-of-mass energies up to $\sqrt{s}=209 \mathrm{GeV}$, Eur. Phys. J. C 46 (2006) 307-341 [hep-ex/0507048].

[8] ATLAS Collaboration, Performance of the reconstruction of large impact parameter tracks in the ATLAS inner detector, ATL-PHYS-PUB-2017-014.

[9] CMS Collaboration, Search for long-lived particles decaying in the CMS endcap muon detectors in proton-proton collisions at $\sqrt{s}=13 \mathrm{TeV}$, CERN-EP-2021-125 [Submitted to Phys. Rev. Lett.].

[10] M. Ester, H.-P. Kriegel, J. Sander, and X. Xu, A density-based algorithm for discovering clusters in large spatial databases with noise, Proceedings of the Second International Conference on Knowledge Discovery and Data Mining Association for the Advancement of Artificial Intelligence (1996) 226.

[11] ATLAS Collaboration, Search for long-lived neutral particles produced in pp collisions at $\sqrt{s}=13 \mathrm{TeV}$ decaying into displaced hadronic jets in the ATLAS inner detector and muon spectrometer, Phys. Rev. D 101 (2020) 052013 [hep-ex/1911. 12575]. 